\title{
Trends of complications and innovative techniques' utilization for colectomies in the United States
}

\author{
Mohamed A. Abd El Aziz ${ }^{1}$ Fabian Grass ${ }^{1} \cdot$ Kevin T. Behm ${ }^{1}$. Sherief Shawki ${ }^{1}$ Anne-Lise D’Angelo ${ }^{1} \cdot$ Kellie L. Mathis ${ }^{1}$. \\ David W. Larson ${ }^{1}$
}

Received: 12 June 2020 / Accepted: 31 July 2020 / Published online: 9 August 2020

(C) Italian Society of Surgery (SIC) 2020

\begin{abstract}
Despite an increasing trend towards utilization of minimally invasive approaches (MIS), results regarding their safety profile are contradictory. All patients who underwent elective colectomy for any underlying disease with an identifiable operative approach available from the targeted colectomy files of the ACS-NSQIP PUFs 2013 to 2018 were included. The trend of utilization and complication rates of the different operative approaches (open, laparoscopic, robotic) were assessed during the inclusion period. Furthermore, overall, surgical, and medical complications were compared between the three approaches. The study cohort included 78,987 patients. Of them, 12,335 (15.6\%) patients underwent open, 57,874 (73.3\%) laparoscopic, and $8,778(11.1 \%)$ robotic surgery. There was an increasing trend towards the utilization of robotic surgery $(2.5 \%$ increase per year) at the expense of the other approaches. With the increasing trend toward the utilization of the robotic approach, a decreasing trend in overall and surgical complications and length of stay was observed. After adjusting for the baseline confounders, robotic surgery was associated with shorter length of stay, lower rate of overall (OR $0.397 ; p<0.05$ compared to open and OR: $0.763 ; p<0.05$ compared to laparoscopy) and surgical complications (OR: $0.464 ; p<0.05$ compared to open and OR: $0.734 ; p<0.05$ compared to laparoscopy). This study revealed an increasing trend toward the utilization of MIS for elective colectomy in the US. Robotic surgery was associated with a decreasing trend in overall and surgical morbidity and length of stay.
\end{abstract}

Keywords Robotic $\cdot$ Laparoscopic $\cdot$ Open $\cdot$ Colectomy $\cdot$ Surgical complications $\cdot$ Trend analysis

\section{Introduction}

Robotic surgery is a minimally invasive technique that is still emerging into mainstay surgical practice [1-3]. The acceptance of a novel surgical technique, such as robotics, premises extensive assessment of safety profiles, along with surgical, functional, and long-term outcomes including quality of life $[1,4-6]$. This assessment will be critical to the greater adoption of robotic surgery [1]. Prior work has evaluated the safety of innovative approaches such as robotics and

Electronic supplementary material The online version of this article (https://doi.org/10.1007/s13304-020-00862-y) contains supplementary material, which is available to authorized users.

David W. Larson

Larson.David2@mayo.edu

1 Division of Colon and Rectal Surgery, Department of Surgery, Mayo Clinic, 200 first St. Southwest, Rochester, MN 55905, USA laparoscopy. These institutional series have shown promising results regarding both short and long term outcomes [7, 8]. Despite these individual efforts, little has been published on a national level regarding robotic surgery utilization and complication rates in the field of colon and rectal surgery.

Colectomy is a common operative procedure performed for both malignant and benign etiologies by general surgeons and colon and rectal surgeons throughout the nation [1,9]. National quality registries, such as the American College of Surgeons National Quality Improvement Program (ACSNSQIP), provide critical data for evaluating the utilization of the operative techniques for elective colectomies [10]. Thus, the aim of this study was to assess national trends of utilization, complications, and length of stay for open, laparoscopic, and robotic elective colectomy using the ACSNSQIP database. 


\section{Materials and methods}

\section{Data source and study population}

We used data from the American College of Surgeons National Surgical Quality Improvement Program (ACSNSQIP) which is a nationally validated, risk-adjusted, and outcome-based program to measure and improve the quality of surgical care [10]. Trained data abstractors from the participant sites use standardized sampling methods and definitions to collect patient-specific and disease-related pre-, intra- and postoperative variables. Patients are identified for inclusion in the final dataset based on a random sample with approximately $20 \%$ of all cases retained [11].

After merging ACS-NSQIP Participant User Files (PUFs) with their corresponding Targeted Colectomies' files, patients who underwent colectomy for any reason between 2013 to 2018 were identified using Current Procedure Terminology (CPT) codes 44140, 44141, 44143, 44144, 44145, 44146, 44147, 44150, 44151, 44155, 44156, 44157, 44158, 44160, 44188, 44204, 44205, 44206, 44207, 44208, 44210, 44211, 44212, 44340, 44345, 44346, 44605, 44626, 45110, 45111, 45112, 45113, 45114, 45116, 45119, 45120, 45121, 45123, 45126, 45136, 45150, 45160, 45395, and 45397. Patients with no operative approach available were excluded using the "COL_APPROACH" variable accessed from the targeted colectomy files. Only patients who had elective, non-urgent colectomy were included in the present study. Patients who had a concurrent operation under the responsibility of another primary surgeon were excluded.

Using the International Classification of Disease Medical Codes (ICD-9-CM and ICD-10-CM), the patients were categorized into three groups: colon cancer (ICD-9-CM; 153.x; and ICD-10-CM; C18.x), inflammatory bowel disease (IBD) (ulcerative colitis; ICD-9-CM: 556.x, and ICD10-CM: K51.x; Crohn's disease: ICD-9-CM: 555.x, and ICD-10-CM: K50.x), and other benign diseases including diverticular disease (ICD-9-CM: 562. $\mathrm{x}$ and ICD-10-CM: K57.x) and benign neoplasms (ICD-9-CM: 211.3 and ICD10-CM: D12.x and K63.5).

Preoperative baseline characteristics, intraoperative, and postoperative information were compared between the three surgical approaches. The trend of the utilization, specific preoperative risk factors (age $>80$ years, Body Mass Index (BMI) $>30 \mathrm{~kg} / \mathrm{m}^{2}$, and American Society of Anesthesiologists (ASA) class $\geq 3$ ), disease category, postoperative complications, and length of stay (LOS) were assessed according to the consecutive years. Postoperative short term (30-day) complications were categorized into overall complication, surgical complication, or medical complication. Postoperative surgical complications included: anastomotic leak, unplanned reoperation, ileus, superficial incisional surgical site infection (SSI), deep SSI, organ space infection, wound disruption, sepsis, septic shock, need for blood transfusion, or death within 30 days after surgery, in line with standardized ACS NSQIP definitions. Post-operative medical complications included: pneumonia, unplanned intubation, pulmonary embolism, deep venous thrombosis, need of mechanical ventilation for more than $48 \mathrm{~h}$, progressive renal insufficiency, urinary tract infection, stroke, myocardial infarction, or cardiac arrest requiring cardiopulmonary resuscitation (CPR).

\section{Statistical analysis}

Data were summarized using frequencies and percentages for categorical variables and median (interquartile range) for continuous variables. Raw proportions (unadjusted) were used to plot the trend graphs. Trend analysis was assessed using linear regression for continuous variables and the Cochran-Armitage test for categorical variables. Fold change for each approach over time was evaluated by dividing the proportional use (or complication rate) of the approach in 2018 by the proportional use of the approach in 2013 . The annual increase or decrease in the proportional use or the complication rate was assessed using linear regression. The coefficient for the consecutive study years, modeled as a continuous variable, was reported as the annual trend. To study the differences between the three surgical approaches, we used the Chi-squared test for categorical variables and the Kruskal-Wallis test for continuous variables. Variables that had an alpha level of less than 0.1 in the univariate analysis were further examined using multivariable binary logistic regression. Ordinal logistic regression was used to assess the impact of the operative approach on the increasing length of stay (LOS), categorized as 0-2 days, 3-4 days, 5-6 days, and $>6$ days, after adjusting for the baseline confounders. The model was fitted using LOS categories as the dependent variable. The proportional odds ratio with its $95 \%$ confidence intervals were reported. Furthermore, a multinominal regression analysis was used to assess the impact of operative approach on the LOS using the lowest LOS category ( $0-2$ days) as a reference. The baseline confounders included in the regression models included; race, age $>80$ years old, $\mathrm{ASA} \geq 3, \mathrm{COPD}, \mathrm{BMI}>30 \mathrm{Kg} / \mathrm{m}^{2}$, ascites, CHF, HTN, currently on dialysis, bleeding disorder, smoking status, functional status, disseminated cancer, chronic steroid use, $>10$ loss of weight before the operation, need a blood transfusion before the operation, systemic sepsis, and operation time. For all the analyses conducted in this study, a $p$-value $<0.05$ was considered statistically significant and all tests were two-sided. Statistical analysis was conducted using the Statistical Package for Social Sciences (SPSS, Version 25; SPSS, Inc., Armonk, NY, USA). 


\section{Results}

In total 78,987 patients were included. Of them, 12,335 (15.6\%) patients had open surgery, 57,874 (73.3\%) had laparoscopic surgery, and $8778(11.1 \%)$ had robotic surgery. The most common indication for surgery was colon cancer ( $47.5 \%$ of operations), followed by benign diseases (diverticular disease or benign neoplasms, $44.85 \%$ ), and followed by IBD (7.6\%). Among all three disease categories, laparoscopy was the most commonly utilized approach (71.1\% for colon cancer, $74.2 \%$ for IBD, and $74.8 \%$ for other benign diseases) (Table 1).

\section{General trends of utilization}

As shown in Fig. 1, the national utilization of the robotic approach for elective non-urgent colectomies has increased from $3.9 \%$ in 2013 to $16 \%$ in 2018 (4.1 fold change; slope,

Table 1 Patients' baseline characteristics

\begin{tabular}{|c|c|c|c|c|c|}
\hline & $\begin{array}{l}\text { Open } \\
N=12,335\end{array}$ & $\begin{array}{l}\text { Laparoscopic } \\
N=57,874\end{array}$ & $\begin{array}{l}\text { Robotic } \\
N=8778\end{array}$ & $\begin{array}{l}\text { Total } \\
N=78,987\end{array}$ & $p$ value \\
\hline Male & $597(48.4 \%)$ & $28,117(48.6 \%)$ & $4331(49.3 \%)$ & $38,419(48.6 \%)$ & 0.357 \\
\hline Race & & & & & $<0.0001$ \\
\hline White & $896(72.7 \%)$ & $42,517(73.5 \%)$ & $7264(82.8 \%)$ & $58,745(74.4 \%)$ & \\
\hline African/American & $1191(9.7 \%)$ & $5080(8.8 \%)$ & $833(9.5 \%)$ & $7104(9.0 \%)$ & \\
\hline Asian & $298(2.4 \%)$ & $1839(3.2 \%)$ & $240(2.7 \%)$ & $2377(3.0 \%)$ & \\
\hline Others & $44(0.4 \%)$ & $374(0.7)$ & $27(0.3 \%)$ & $445(0.5 \%)$ & \\
\hline Unknown & $1838(14.9 \%)$ & $8064(13.9 \%)$ & $414(4.7 \%)$ & $10,316(13.1 \%)$ & \\
\hline Age $>80$ years & $1747(14.2 \%)$ & $5231(9.0 \%)$ & $555(6.3 \%)$ & $7533(9.5 \%)$ & $<0.0001$ \\
\hline $\mathrm{BMI}>30 \mathrm{~kg} / \mathrm{m}^{2}$ & $4360(35.5 \%)$ & $21,357(37.1 \%)$ & $3662(41.8 \%)$ & $29,379(37.4 \%)$ & $<0.0001$ \\
\hline $\mathrm{ASA} \geq 3$ & $7748(62.8 \%)$ & $28,112(48.6 \%)$ & $4296(48.9 \%)$ & $40,156(50.8 \%)$ & $<0.0001$ \\
\hline $\mathrm{DM}$ & $2277(18.5 \%)$ & $9116(15.8 \%)$ & $1451(16.5 \%)$ & $12,844(16.3 \%)$ & $<0.0001$ \\
\hline COPD & $794(6.4 \%)$ & $2428(4.2 \%)$ & $352(4.0 \%)$ & $3574(4.5 \%)$ & $<0.0001$ \\
\hline Ascites & $48(0.4 \%)$ & $76(0.1 \%)$ & $7(0.1 \%)$ & $131(0.2 \%)$ & $<0.0001$ \\
\hline $\mathrm{CHF}$ & $149(1.2 \%)$ & $313(0.5 \%)$ & $39(0.4 \%)$ & $501(0.6 \%)$ & $<0.0001$ \\
\hline HTN requiring medication & $6478(52.5 \%)$ & $27,892(48.2 \%)$ & $4431(50.5 \%)$ & $38,801(49.1 \%)$ & $<0.0001$ \\
\hline Hemodialysis & $90(0.7 \%)$ & $271(0.5 \%)$ & $34(0.4 \%)$ & $395(0.5 \%)$ & $<0.0001$ \\
\hline Bleeding disorder & $390(3.2 \%)$ & $1140(2.0 \%)$ & $171(1.9 \%)$ & $1701(2.2 \%)$ & $<0.0001$ \\
\hline Number of comorbidities & & & & & $<0.0001$ \\
\hline No comorbidities & $5094(41.3 \%)$ & $27,173(47.0 \%)$ & $3932(44.8 \%)$ & $36,199(45.8 \%)$ & \\
\hline One & $4678(37.9 \%)$ & $21,280(36.8 \%)$ & $3355(38.2 \%)$ & $29,313(37.1 \%)$ & \\
\hline Two & $2187(17.7 \%)$ & $8390(14.5 \%)$ & $1352(15.4 \%)$ & $11,929(15.1 \%)$ & \\
\hline Three & $331(2.7 \%)$ & $951(1.6 \%)$ & $131(1.5 \%)$ & $1413(1.8 \%)$ & \\
\hline Four & $44(0.4 \%)$ & $77(0.1 \%)$ & $7(0.1 \%)$ & $128(0.2 \%)$ & \\
\hline Five & 1 & 3 & $1(0.0 \%)$ & $5(0.0 \%)$ & \\
\hline Post-operative diagnosis & & & & & $<0.0001$ \\
\hline Colon cancer & $7014(56.9 \%)$ & $26,896(46.5 \%)$ & $3617(41.2 \%)$ & $37,527(47.5 \%)$ & \\
\hline IBD & $1319(10.7 \%)$ & $4474(7.7 \%)$ & $239(2.7 \%)$ & $6032(7.6 \%)$ & \\
\hline Others* & $4002(32.4 \%)$ & $26,504(45.8 \%)$ & $4922(56.1 \%)$ & $35,428(44.9 \%)$ & \\
\hline Functional status: independent & $12,004(97.3 \%)$ & $56,990(98.5 \%)$ & $8627(98.3 \%)$ & $77,621(98.3 \%)$ & $<0.0001$ \\
\hline Disseminated cancer & $979(7.9 \%)$ & $1640(2.8 \%)$ & $188(2.1 \%)$ & $2807(3.6 \%)$ & $<0.0001$ \\
\hline Chronic steroid use & $1330(10.8 \%)$ & $4598(7.9 \%)$ & $408(4.6 \%)$ & $6336(8.0 \%)$ & $<0.0001$ \\
\hline$>10 \%$ loss of body weight in last 6 months & $612(5.0 \%)$ & $1603(2.8 \%)$ & $165(1.9 \%)$ & $2380(3.0 \%)$ & $<0.0001$ \\
\hline Transfusion of $\geq 1 \mathrm{pRBCs}$ in $72 \mathrm{~h}$ before surgery & $169(1.4 \%)$ & $296(0.5 \%)$ & $22(0.3 \%)$ & $487(0.6 \%)$ & $<0.0001$ \\
\hline Operation time; median (IQR); minutes & $134(96-189)$ & $151(114-201)$ & $194(152-247)$ & & $<0.0001$ \\
\hline
\end{tabular}

$B M I$ body mass index, ASA American Society of Anesthesiologists, DM diabetes mellitus, COPD chronic obstructive pulmonary disease, $C H F$ congestive heart failure, $H T N$ hypertension, $I B D$ inflammatory bowel disease, $p R B C s$ packed red blood cells, $I Q R$ interquartile range

*Others: including diverticular diseases or benign conditions of the colon 

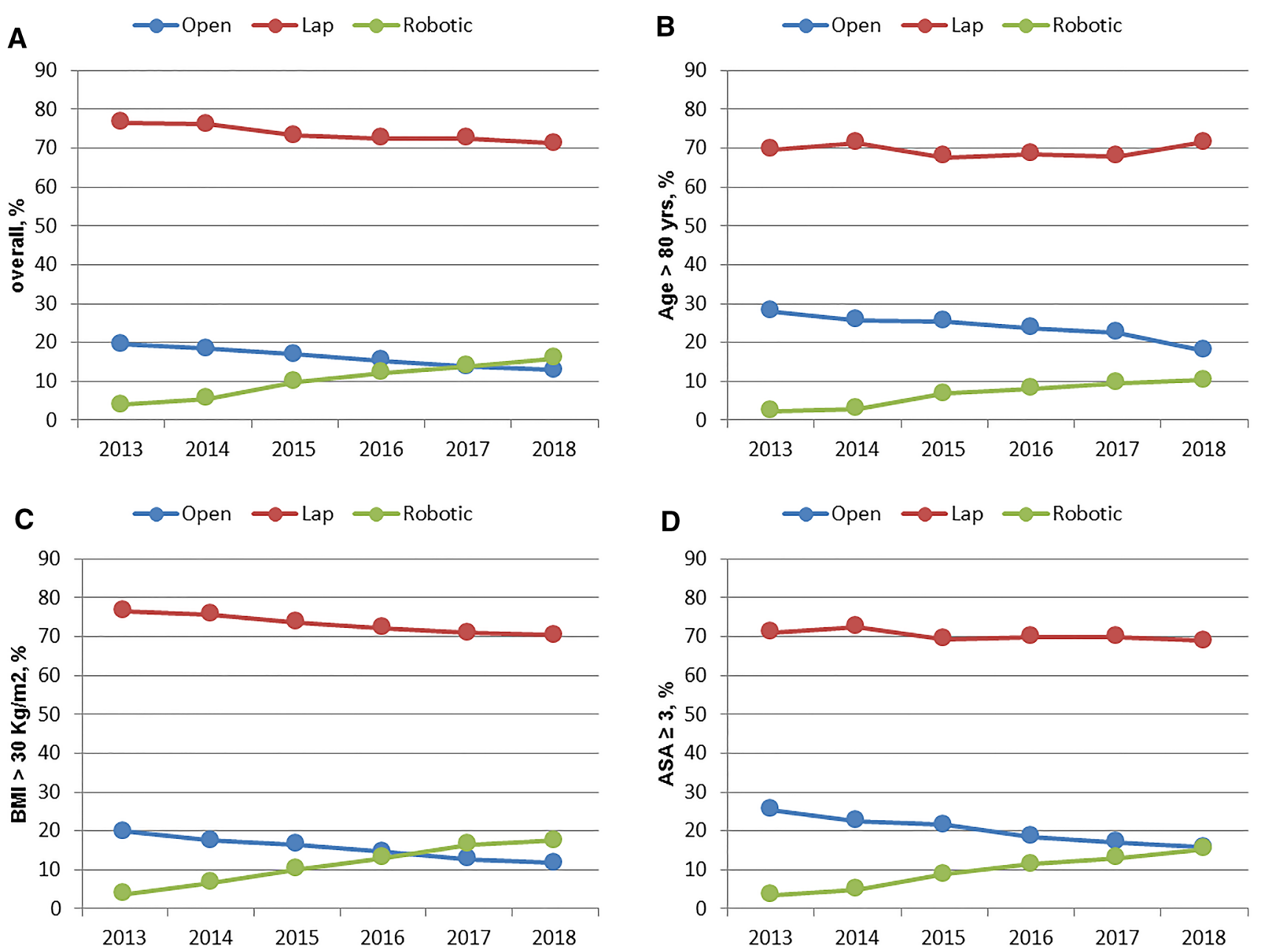

Fig. 1 Trend utilization according to operative approach. a Overall trend: robotic approach for elective non-urgent colectomies has increased from 3.9\% in 2013 to $16 \%$ in 2018 (4.1 fold change; slope, $2.5 \%$ per year; $95 \%$ CI $2.3-2.6 \%$ ) at the expense of a decrease in the utilization of open (19.5\% in 2013 to $12.9 \%$ in 2018; 0.67 fold change; slope, $-1.4 \%$ per year; $95 \%$ CI $-1.6 \%$ to $-1.2 \%$ ) and laparoscopic (76.6\% in 2013 to $71.2 \%$ in 2018; 0.9 fold change; slope $-1.1 \%$ per year, $95 \%$ CI -1.2 to -0.9 ) approaches; ( $p$-value <0.0001). b Age > 80-years-old: the utilization of the robotic approach increased from $2.4 \%$ in 2013 to $10.4 \%$ in 2018 (4.3 fold change, slope $1.7 \%$ per year, $95 \%$ CI $1.4-2.1 \%$ ) at the expense of the open approach which decreased from $27.9 \%$ in 2013 to $18 \%$ in 2018 (0.65 fold change, slope $-1.8 \%$ per year, $95 \%$ CI $-2.4 \%$ to $-1.3 \%$ ). c $\mathrm{BMI}>30 \mathrm{Kg} / \mathrm{m}^{2}$ : robotic approach utilization has increased among

$2.5 \%$ per year; $95 \%$ CI $2.3-2.6 \%$ ) at the expense of open (19.5\% in 2013 to $12.9 \%$ in 2018 ; 0.67 fold change; slope, $-1.4 \%$ per year; $95 \%$ CI $-1.6 \%$ to $-1.2 \%$ ) and laparoscopic $(76.6 \%$ in 2013 to $71.2 \%$ in 2018 ; 0.9 fold change; slope $-1.1 \%$ per year, $95 \%$ CI -1.2 to -0.9$)$ approaches; ( $p$-value $<0.0001)$. A similar trend was observed for the subgroup analysis according to disease category (Supplementary Fig. 1).

\section{Subgroup analysis of high-risk patients}

Among older patients ( $>80$ years), the utilization of the robotic approach increased at the expense of the open approach. Furthermore, robotic utilization has increased among patients with obesity (BMI $>30 \mathrm{~kg} / \mathrm{m}^{2}$ ) and ASA class $\geq 3$ at the expense of both open and laparoscopic approaches (Fig. 1). 


\section{Trends of postoperative morbidity}

\section{Overall complications}

Open surgery was associated with a higher overall complication rate (34.4\%) compared to laparoscopic (19.7\%) and robotic $(16.8 \%)$ approaches $(p$-value $<0.0001)$. After adjusting for baseline confounders, robotic surgery was associated with a lower overall complication rate compared to both open (OR: 0.397; 95\% CI [0.370-0.426]) and laparoscopic (OR 0.763; 95\% CI [0.717-0.812]) surgery. Furthermore, the laparoscopic approach was associated with a lower overall complication rate compared to the open approach (OR 0.521; 95\% CI [0.499-0.545]) (Tables 2 and 3).

Table 2 Postoperative complications

\begin{tabular}{|c|c|c|c|c|c|}
\hline & $\begin{array}{l}\text { Open } \\
N=12,335\end{array}$ & $\begin{array}{l}\text { Laparoscopic } \\
N=57,874\end{array}$ & $\begin{array}{l}\text { Robotic } \\
N=8778\end{array}$ & $\begin{array}{l}\text { Total } \\
N=78,987\end{array}$ & $p$ value \\
\hline Overall complications & $4247(34.4 \%)$ & $11,421(19.7 \%)$ & $1479(16.8 \%)$ & $17,147(21.7 \%)$ & $<0.0001$ \\
\hline Surgical complications & $3860(31.4 \%)$ & $10,148(17.6 \%)$ & $1273(14.6 \%)$ & $15,281(19.4 \%)$ & $<0.0001$ \\
\hline Medical complications & $1020(8.3 \%)$ & $2578(4.5 \%)$ & $378(4.3 \%)$ & $3976(5.0 \%)$ & $<0.0001$ \\
\hline Unplanned conversion to open & NA & $5382(9.3 \%)$ & $448(5.1 \%)$ & $5830(7.4 \%)$ & $<0.0001$ \\
\hline \multicolumn{6}{|l|}{ SSI } \\
\hline Superficial SSI & $663(5.4 \%)$ & $1651(2.9 \%)$ & $191(2.2 \%)$ & $2505(3.2 \%)$ & $<0.0001$ \\
\hline Deep SSI & $136(1.1 \%)$ & $223(0.4 \%)$ & $31(0.4 \%)$ & $390(0.5 \%)$ & $<0.0001$ \\
\hline Organ/space SSI & $607(4.9 \%)$ & $1668(2.9 \%)$ & $250(2.8 \%)$ & $2525(3.2 \%)$ & $<0.0001$ \\
\hline Any SSI & $1342(10.9 \%)$ & $3420(5.9 \%)$ & $448(5.1 \%)$ & $5210(6.6 \%)$ & $<0.0001$ \\
\hline Wound disruption & $123(1.0 \%)$ & $231(0.4 \%)$ & $22(0.3 \%)$ & $376(0.5 \%)$ & $<0.0001$ \\
\hline \multicolumn{6}{|l|}{ Systemic sepsis } \\
\hline Sepsis & $401(3.3 \%)$ & $1030(1.8 \%)$ & $130(1.5 \%)$ & $1561(2.0 \%)$ & $<0.0001$ \\
\hline Septic shock & $195(1.6 \%)$ & $391(0.7 \%)$ & $51(0.6 \%)$ & $637(0.8 \%)$ & $<0.0001$ \\
\hline \multicolumn{6}{|l|}{ Other surgical complications } \\
\hline Need for blood transfusion & $1135(9.2 \%)$ & $2707(4.7 \%)$ & $319(3.6 \%)$ & $4161(5.3 \%)$ & $<0.0001$ \\
\hline Ileus & $2066(16.8 \%)$ & $5066(8.8 \%)$ & $625(7.1 \%)$ & $7757(9.8 \%)$ & $<0.0001$ \\
\hline Unplanned reoperation & $566(4.6 \%)$ & $1863(3.2 \%)$ & $333(3.8 \%)$ & $2762(3.5 \%)$ & $<0.0001$ \\
\hline Leak & $428(3.5 \%)$ & $1406(2.4 \%)$ & $195(2.2 \%)$ & $2029(2.6 \%)$ & $<0.0001$ \\
\hline \multicolumn{6}{|l|}{ Respiratory complications } \\
\hline Pneumonia & $276(2.2 \%)$ & $624(1.1 \%)$ & $67(0.8 \%)$ & $967(1.2 \%)$ & $<0.0001$ \\
\hline Unplanned intubation & $222(1.8 \%)$ & $444(0.8 \%)$ & $53(0.6 \%)$ & $719(0.9 \%)$ & $<0.0001$ \\
\hline On ventilator for $>48 \mathrm{~h}$ & $155(1.3 \%)$ & $306(0.5 \%)$ & $45(0.5 \%)$ & $506(0.6 \%)$ & $<0.0001$ \\
\hline \multicolumn{6}{|l|}{ VTE } \\
\hline $\mathrm{PE}$ & $84(0.7 \%)$ & $211(0.4 \%)$ & $32(0.4 \%)$ & $327(0.4 \%)$ & $<0.0001$ \\
\hline DVT & $164(1.3 \%)$ & $451(0.8 \%)$ & $68(0.8 \%)$ & $683(0.9 \%)$ & $<0.0001$ \\
\hline \multicolumn{6}{|l|}{ Renal complications } \\
\hline Progressive renal insufficiency & $99(0.8 \%)$ & $184(0.3 \%)$ & $47(0.5 \%)$ & $330(0.4 \%)$ & $<0.0001$ \\
\hline UTI & $254(2.1 \%)$ & $735(1.3 \%)$ & $112(1.3 \%)$ & $1101(1.4 \%)$ & $<0.0001$ \\
\hline Stroke & $35(0.3 \%)$ & $90(0.2 \%)$ & $18(0.2 \%)$ & $143(0.2 \%)$ & 0.01 \\
\hline \multicolumn{6}{|l|}{ Cardiovascular complications } \\
\hline Cardiac arrest requiring CPR & $67(0.5 \%)$ & $149(0.3 \%)$ & $26(0.3 \%)$ & $242(0.3 \%)$ & $<0.0001$ \\
\hline MI & $110(0.9 \%)$ & $273(0.5 \%)$ & $30(0.3 \%)$ & $413(0.5 \%)$ & $<0.0001$ \\
\hline LOS: days & & & & & $<0.0001$ \\
\hline $0-2$ & $507(4.1 \%)$ & $11,076(19.2 \%)$ & $2650(30.2 \%)$ & $14,233(18.0 \%)$ & \\
\hline $3-4$ & $3747(30.4 \%)$ & $28,161(48.7 \%)$ & $4129(47.1 \%)$ & $36,037(45.7 \%)$ & \\
\hline $5-6$ & $3753(30.5 \%)$ & $10,367(17.9 \%)$ & $1138(13.0 \%)$ & $15,258(19.3 \%)$ & \\
\hline$>6$ & $4302(35.0 \%)$ & $8206(14.2 \%)$ & $853(9.7 \%)$ & $13,361(16.9 \%)$ & \\
\hline 30 day mortality & $170(1.4 \%)$ & $299(0.5 \%)$ & $39(0.4 \%)$ & $508(0.6 \%)$ & $<0.0001$ \\
\hline
\end{tabular}

SSI surgical site infection, VTE vascular thromboembolism, $P E$ pulmonary embolism, DVT deep venous thrombosis, $U T I$ urinary tract infection, $C P R$ cardiopulmonary resuscitation, $M I$ myocardial infarction, $L O S$ length of stay 
Table 3 Multivariable regression analysis adjusting for the baseline confounders

\begin{tabular}{|c|c|c|c|c|c|c|}
\hline & \multicolumn{2}{|c|}{ Laparoscopic versus open } & \multicolumn{2}{|l|}{ Robotic versus open } & \multicolumn{2}{|c|}{ Robotic versus laparoscopic } \\
\hline & OR $(95 \% \mathrm{CI})$ & $p$ value & OR $(95 \% \mathrm{CI})$ & $p$ value & OR $(95 \% \mathrm{CI})$ & $p$ value \\
\hline Overall complications & $0.521(0.499-0.545)$ & $<0.0001$ & $0.397(0.370-0.426)$ & $<0.0001$ & $0.763(0.717-0.812)$ & $<0.0001$ \\
\hline Surgical complications & $0.543(0.519-0.568)$ & $<0.0001$ & $0.464(0.431-0.499)$ & $<0.0001$ & $0.734(0.687-0.784)$ & $<0.0001$ \\
\hline Medical complications & $0.516(0.493-0.540)$ & $<0.0001$ & $0.377(0.350-0.406)$ & $<0.0001$ & $0.926(0.825-1.038)$ & 0.187 \\
\hline Unplanned conversion to open & NA & & NA & & $0.551(0.498-0.610)$ & $<0.0001$ \\
\hline \multicolumn{7}{|l|}{ SSI } \\
\hline Superficial SSI & $0.544(0.495-0.598)$ & $<0.0001$ & $0.432(0.366-0.510)$ & $<0.0001$ & $0.693(0.594-0.809)$ & $<0.0001$ \\
\hline Deep SSI & $0.369(0.296-0.459)$ & $<0.0001$ & $0.321(0.215-0.480)$ & $<0.0001$ & $0.822(0.560-1.207)$ & 0.318 \\
\hline Organ/space SSI & $0.615(0.558-0.679)$ & $<0.0001$ & $0.563(0.482-0.658)$ & $<0.0001$ & $0.903(0.786-1.038)$ & 0.150 \\
\hline Any SSI & $0.533(0.498-0.571)$ & $<0.0001$ & $0.420(0.374-0.470)$ & $<0.0001$ & $0.775(0.698-0.860)$ & $<0.0001$ \\
\hline Wound disruption & $0.481(0.382-0.604)$ & $<0.0001$ & $0.280(0.176-0.446)$ & $<0.0001$ & $0.551(0.353-0.860)$ & 0.009 \\
\hline \multicolumn{7}{|l|}{ Systemic sepsis } \\
\hline Sepsis & $0.640(0.566-0.724)$ & $<0.0001$ & $0.486(0.395-0.597)$ & $<0.0001$ & $0.753(0.624-0.909)$ & 0.003 \\
\hline Septic shock & $0.512(0.428-0.611)$ & $<0.0001$ & $0.418(0.304-0.574)$ & $<0.0001$ & $0.812(0.602-1.096)$ & 0.173 \\
\hline \multicolumn{7}{|l|}{ Other surgical complications } \\
\hline Need for blood transfusion & $0.618(0.573-0.667)$ & $<0.0001$ & $0.527(0.462-0.6)$ & $<0.0001$ & $0.858(0.760-0.968)$ & 0.013 \\
\hline Ileus & $0.547(0.517-0.579)$ & $<0.0001$ & $0.461(0.418-0.507)$ & $<0.0001$ & $0.846(0.775-0.923)$ & $<0.0001$ \\
\hline Unplanned reoperation & $0.768(0.696-0.848)$ & $<0.0001$ & $0.864(0.748-0.997)$ & 0.045 & $1.103(0.976-1.246)$ & 0.117 \\
\hline Leak & $0.735(0.657-0.823)$ & $<0.0001$ & $0.637(0.534-0.761)$ & $<0.0001$ & $0.853(0.730-0.997)$ & 0.046 \\
\hline \multicolumn{7}{|l|}{ Respiratory complications } \\
\hline Pneumonia & $0.613(0.528-0.711)$ & $<0.0001$ & $0.434(0.329-0.573)$ & $<0.0001$ & $0.699(0.539-0.907)$ & 0.007 \\
\hline Unplanned intubation & $0.544(0.460-0.644)$ & $<0.0001$ & $0.411(0.301-0.561)$ & $<0.0001$ & $0.771(0.574-1.034)$ & 0.082 \\
\hline On ventilator for $>48 \mathrm{hrs}$ & $0.529(0.432-0.647)$ & $<0.0001$ & $0.483(0.342-0.681)$ & $<0.0001$ & $0.941(0.681-1.300)$ & 0.714 \\
\hline \multicolumn{7}{|l|}{ VTE } \\
\hline $\mathrm{PE}$ & $0.581(0.448-0.754)$ & $<0.0001$ & $0.523(0.344-0.796)$ & 0.002 & $0.853(0.583-1.247)$ & 0.411 \\
\hline DVT & $0.658(0.546-0.791)$ & $<0.0001$ & $0.596(0.445-0.799)$ & 0.001 & $0.889(0.684-1.156)$ & 0.380 \\
\hline \multicolumn{7}{|l|}{ Renal complications } \\
\hline Progressive renal insufficiency & $0.478(0.372-0.615)$ & $<0.0001$ & $0.699(0.487-1.004)$ & 0.053 & $1.464(1.051-2.040)$ & 0.024 \\
\hline UTI & $0.701(0.604-0.814)$ & $<0.0001$ & $0.721(0.571-0.909)$ & 0.006 & $1.031(0.839-1.267)$ & 0.772 \\
\hline Stroke & $0.725(0.485-1.082)$ & 0.115 & $0.987(0.545-1.788)$ & 0.966 & $1.394(0.823-2.360)$ & 0.217 \\
\hline \multicolumn{7}{|l|}{ Cardiovascular complications } \\
\hline Cardiac arrest requiring CPR & $0.608(0.451-0.820)$ & 0.001 & $0.736(0.459-1.182)$ & 0.205 & $1.232(0.800-1.899)$ & 0.343 \\
\hline MI & $0.649(0.517-0.817)$ & $<0.0001$ & $0.483(0.318-0.733)$ & 0.001 & $0.712(0.483-1.049)$ & 0.086 \\
\hline \multicolumn{7}{|l|}{ LOS, days } \\
\hline $0-2$ & Reference & & Reference & & Reference & \\
\hline $3-4$ & $0.321(0.291-0.353)$ & $<0.0001$ & $0.162(0.146-0.181)$ & $<0.0001$ & $0.503(0.475-0.532)$ & $<0.0001$ \\
\hline $5-6$ & $0.115(0.104-0.127)$ & $<0.0001$ & $0.038(0.034-0.043)$ & $<0.0001$ & $0.326(0.301-0.353)$ & $<0.0001$ \\
\hline$>6$ & $0.08(0.073-0.089)$ & $<0.0001$ & $0.022(0.020-0.025)$ & $<0.0001$ & $0.278(0.254-0.304)$ & $<0.0001$ \\
\hline 30 day mortality & $0.541(0.444-0.660)$ & $<0.0001$ & $0.521(0.362-0.750)$ & $<0.0001$ & $0.956(0.675-1.352)$ & 0.798 \\
\hline
\end{tabular}

SSI surgical site infection, VTE vascular thromboembolism, $P E$ pulmonary embolism, DVT deep venous thrombosis, UTI urinary tract infection, $C P R$ cardiopulmonary resuscitation, $M I$ myocardial infarction, $L O S$ length of stay

Over the six study years, the overall complication rate decreased significantly among all three operative approaches (Fig. 2).

\section{Surgical complications}

Open surgeries had a higher overall surgical complication rate $(31.4 \%)$ compared to laparoscopic $(17.6 \%)$ and robotic $(14.6 \%)$ approaches $(p$-value $<0.0001)$. After adjusting for 
A $\multimap$ Open $\multimap$ Lap $\multimap$ Robotic

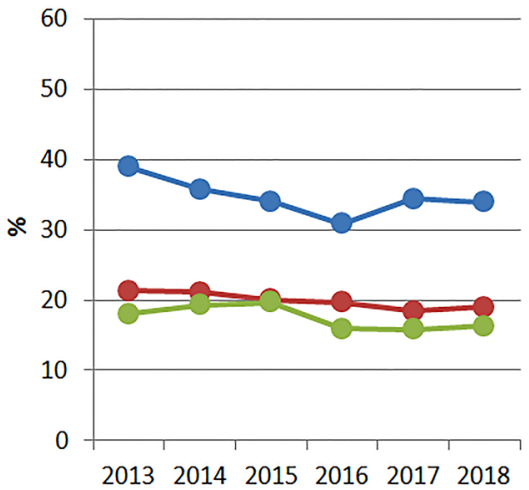

B

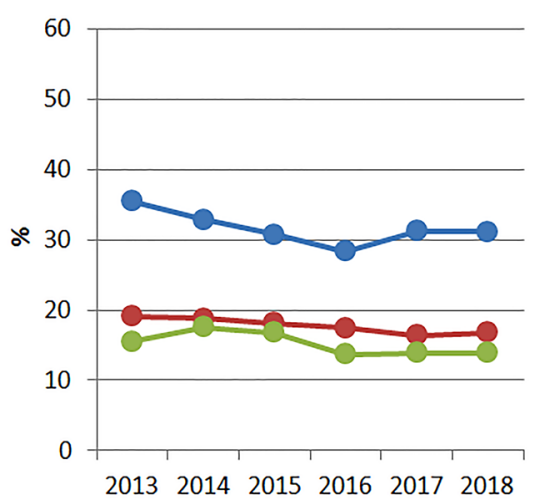

C $\rightarrow$ Open $\rightarrow$ Lap $\multimap$ Robotic

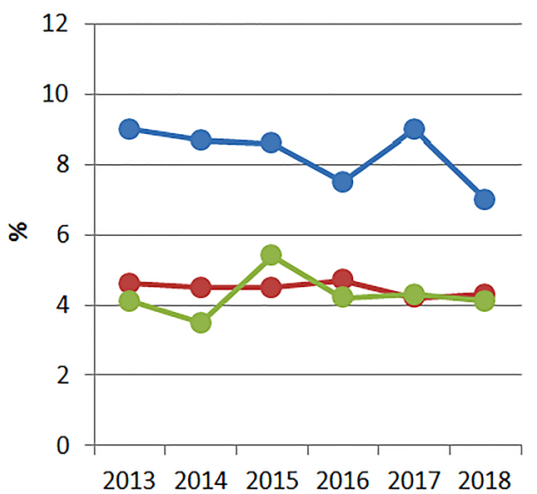

Fig. 2 Trends of postoperative complications according to operative approach. a Overall complications: the overall complication rate decreased over time for the three operative approaches; open (38.9\% in 2013 to $33.9 \%$ in $2018,0.87$ fold change, slope $-0.9 \%$ per year, $95 \%$ CI [ $-1.4 \%$ to $-0.4 \%$ ] $p$-value 0.001$)$, laparoscopic (21.3\% in 2013 to $19 \%$ in $2018,0.89$ fold change, slope $-0.6 \%$ per year, 95\% CI [ $-0.8 \%$ to $-0.4 \%$ ] $p$-value $<0.0001$ ), and robotic (18\% in 2013 to $16.3 \%$ in $2018,0.9$ fold change, slope $-0.7 \%$ per year, $95 \%$ CI $[-1.3 \%$ to $-0.2 \%$ ], $p$ value 0.012 ). b Surgical complications: the surgical complication rate decreased over time for the three operative approaches; open $(35.5 \%$ in 2013 to $31.1 \%$ in 2018, 0.88 fold change, slope $-0.8 \%$ per year, $95 \%$ CI $[-1.3 \%$ to

baseline confounders, robotic surgery was associated with a lower overall surgical complication rate compared to open (OR: 0.464; 95\% CI [0.431-0.499]) and laparoscopic (OR $0.734 ; 95 \%$ CI [0.687-0.784]) approaches. Furthermore, the laparoscopic approach was associated with a lower overall surgical complication rate comparing to the open approach (OR $0.543 ; 95 \%$ CI [0.519-0.568]), as detailed in (Tables 2 and 3).

Over the inclusion period, the overall surgical complication rate decreased significantly among the three operative approaches (Fig. 2).

\section{Medical complications}

Open surgeries had a higher overall medical complication rate $(8.3 \%)$ compared to laparoscopic $(4.5 \%)$ and robotic $(4.3 \%)$ approaches $(p$-value $<0.0001)$. After adjusting for baseline confounders, robotic surgery was associated with a lower overall medical complication rate compared to open (OR: $0.377 ; 95 \%$ CI [0.350-0.406]) but not laparoscopic (OR 0.926; 95\% CI [0.825-1.038]) approaches. Furthermore, the laparoscopic approach was associated with a lower risk of overall medical complication rate comparing to the open approach (OR 0.516; 95\% CI [0.493-0.540]), as detailed in (Tables 2 and 3).
$-0.3 \%$ ], $p$-value $=0.002)$, laparoscopic $(19.1 \%$ in 2013 to $16.8 \%$ in 2018, 0.88 fold change, slope $-0.5 \%$ per year, $95 \%$ CI $[-0.7 \%$ to $-0.4 \%$ ], $p$-value $<0.0001$ ), and robotic $(15.5 \%$ in 2013 to $13.9 \%$ in 2018, 0.9 fold change, slope $-0.7 \%$ per year, $95 \%$ CI $[-1.2 \%$ to - 0.2], $p$ value $=0.008)$ c Medical complications: the medical complication rate decreased over time for the open approach (9\% in 2013 to $7 \%$ in $2018,0.8$ fold change, slope $-0.3 \%$ per year, $95 \%$ CI [ -0.6 to -0.0001$], p$ value $=0.041)$ but not for the laparoscopic $(4.6 \%$ in 2013 to $4.3 \%$ in $2018,0.9$ fold change, slope $-0.1 \%$ per year, $95 \%$ $\mathrm{CI}[-0.2$ to -0.0004$], p$ value $=0.239)$ and robotic $(4.1 \%$ in 2013 to $4.1 \%$ in 2018 , onefold change, slope $-0.1,95 \%$ CI [ -0.4 to 0.002 ], $p$ value $=0.585)$ approaches

Over the inclusion period, the overall medical complication rate decreased significantly among open surgeries but not among robotic and laparoscopic surgeries (Fig. 2).

\section{Trend analysis of length of stay (LOS)}

Robotic surgery resulted in a shorter length of stay than open and laparoscopic surgeries (as summarized in Table 2) even after adjusting for baseline confounders as the ordinal regression showed the odds ratio for increasing LOS were -2.067 ; $95 \%$ CI [ -2.121 to -2.012$]$ for robotic versus open, $-1.315 ; 95 \%$ CI [ -1.353 to - 1.277] for laparoscopic versus open, and $-0.753 ; 95 \%$ CI [ -0.797 to -0.709$]$ for robotic versus laparoscopic. Results for the multinominal regression are presented in Table 3.

Most of the patients who had robotic surgery were discharged by the third or fourth postoperative days $(47.1 \%)$ or within the first two days (30.2\%). The proportion of patients who discharged within the first two days after robotic surgery increased over time (16\% in 2013 to $38.2 \%$ in 2018; 2.3 fold change, slope 5\% per year, 95\% CI 4.3-5.7\%) (Fig. 3).

A similar trend was noted for laparoscopic surgery (Fig. 3). Trends for the open approach are described in detail in Fig. 3.

Detailed tables of trends and complications are provided in supplementary Tables 1-7. 

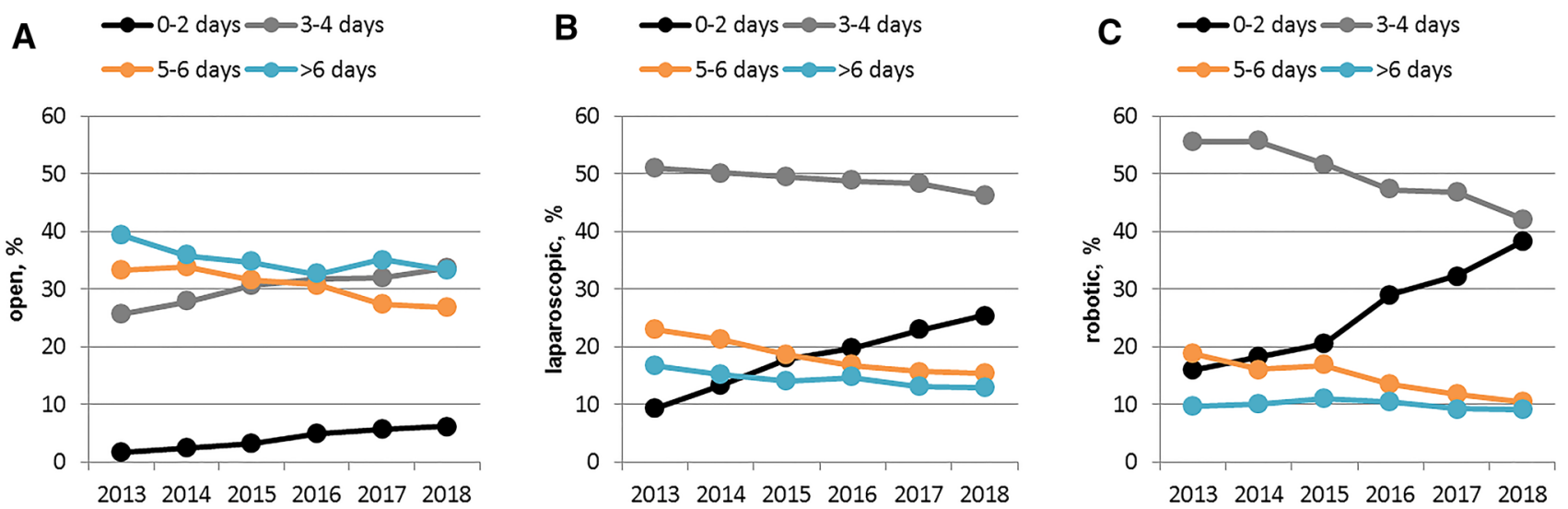

Fig. 3 Trends of length of stay according to operative approach. 0-2 days: trend in increasing the proportion of patients discharged in the first two days across all approaches, a Open: (1.6\% in 2013 to $6.2 \%$ in $2018,3.9$ fold change, slope $1 \%$ per year, $95 \%$ CI [0.8$1.2 \%$ ], $p$-value $<0.0001)$, b Laparoscopic: $(9.3 \%$ in 2013 to $25.4 \%$ in 2018, 2.7 fold change, slope $3.1 \%$ per year, 95\% CI [2.9-3.3\%], $p$-value < 0.0001), c Robotic: (16\% in 2013 to $38.2 \%$ in 2018, 2.3 fold change, slope $5 \%$ per year, $95 \%$ CI $[4.3-5.7 \%], p$-value $<0.0001)$. 3-4 days: increasing trend for the a open approach (25.7\% in 2013 to $33.7 \%$ in $2018,1.3$ fold change, slope $1.5 \%$ per year, $95 \%$ CI [ $1 \%$ to $2 \%$, p value $<0.0001)$. Decreasing trend for the b laparoscopic $(51 \%$ in 2013 to $46.3 \%$ in $2018,0.9$ fold change, slope $-0.9 \%$ per year, $95 \%$ CI $[-1.1 \%$ to $-0.6 \%] p$-value $<0.0001)$ and $\mathbf{c}$ robotic $(55.5 \%$ in 2013 to $42.2 \%$ in $2018,0.76$ fold change, slope $-2.9 \%$ per year, $95 \%$ CI $[-3.6 \%$ to $-2.2 \%$ ], $p$ value $<0.0001$ ) approach. 5-6 days: a decreasing trend for all the operative approaches, a Open: $33.3 \%$ in 2013 to $26.9 \%$ in $2018,0.8$ fold change, slope $-1.5 \%$ per year, $95 \%$ CI [ $-2 \%$ to $-1 \%$ ], $p$-value $<0.0001$, b laparoscopic: $23 \%$ in 2013 to $15.4 \%$ in $2018,0.67$ fold change, slope $-1.5 \%$ per year, $95 \% \mathrm{CI}$ [ $-1.7 \%$ to $-1.4 \%], p$-value $<0.0001$, and c robotic: $18.9 \%$ in 2013 to $10.5 \%$ in $2018,0.56$ fold change, slope $-1.7 \%$ per year, $95 \% \mathrm{CI}$ [ $-2.2 \%$ to -1.2$],$ p-value $<0.0001 .>6$ days: a decreasing trend for all the operative approaches, a Open: $39.4 \%$ in 2013 to $33.2 \%$ in $2018,0.8$ fold change, slope $-1 \%$ per year, $95 \%$ CI [ $-1.5 \%$ to - $0.5 \%$ ], $p$-value $<0.0001$, b laparoscopic: $16.7 \%$ in 2013 to $12.9 \%$ in $2018,0.77$ fold change, slope $-0.7 \%$ per year, $95 \%$ CI [ $-0.9 \%$ to $-0.5 \%$ ], $p$-value $<0.0001$, and $\mathbf{c}$ robotic: $9.6 \%$ in 2013 to $9.1 \%$ in $2018,0.95$ fold change, slope $-0.4 \%$ per year, $95 \%$ CI $[-0.8 \%$ to $0.001], p$-value $=0.097$

directly OR through all 3 platforms could not be definitely elucidated.

A recent study from a nationally representative sample of Medicare beneficiaries in the U.S. reported a similar increasing trend towards the adoption of the robotic platform for elective colectomy between 2010 and 2016 [1]. Interestingly, the proportion of patients who underwent open colectomy was higher than what has been found in our study, which raises the question of a potential selection bias favoring open surgery in higher-risk Medicare patients. This former study used a robust risk-adjustment method (instrumental variable analysis) to account for unmeasured confounders and found that the robotic platform was associated with a lower risk of postoperative complications compared to open surgery in high volume but not low to average volume centers [1]. However, there was no statistically significant benefit of the robotic approach over the laparoscopic approach even in high volume centers [1]. Although in our study we have found a similar potential benefit of the robotic platform over both open and laparoscopic approaches, case-volume percenter could not be adjusted for as this information is not provided in ACS-NSQIP.

Data from recent randomized controlled trials showed ambiguous results regarding the potential benefit of robotic 
surgery. While safety profiles between laparoscopic and robotic approaches for colectomy appeared to be similar, a clear benefit of the robotic approach could not be demonstrated by the landmark ROLARR trial [12]. Nevertheless, our findings are aligned with data of recent systematic reviews revealing reduced anastomotic complications, conversion rate, and length of stay with robotic surgery compared to the laparoscopic approach [13-15]. Moreover, in cases with more challenging anatomy than colectomy such as rectal cancer cases, robotic surgery could provide benefits beyond the laparoscopic approach when implemented in a high-volume practice $[8,16-18]$.

The decreasing complication rate over time in patients who underwent open surgery might reflect the widespread implementation of enhanced recovery protocols and improved surgical and perioperative care in general, potentially also reflecting better conditioning of patients through prehabilitation programs $[19,20]$. The combination of evidence-based perioperative care and well-structured training programs for minimally invasive surgeries bears an important potential to improve surgical outcomes further [19-24].

\section{Limitations}

The results of this study have to be interpreted with caution considering limitations related to ACS-NSQIP including a high risk of selection bias regarding patients and operative approach given a wide heterogeneity of practice and experience of contributing surgeons. This was accounted for through risk-stratification and -adjustment. The studied sample represents only $20 \%$ of the total number of patients in the U.S. and the targeted file does not capture all patients for each year. Furthermore, ACSNSQIP provides data on short term (30-day) morbidity only, whereas long term outcomes were not available. Lastly, a sensitivity analysis according to the volume of robotic cases per hospital could not be performed as this information is not provided.

\section{Conclusion}

The adoption of robotic surgery is increasing in the U.S. for elective colectomy and is associated with a decrease of postoperative complications and length of stay. Robotic surgery appears to be independently associated with a lower rate of short-term morbidity and surgical conversion compared to both open and laparoscopic approaches.

Funding This research did not receive any specific grant from funding agencies in the public, commercial, or non profit sectors.

\section{Compliance with ethical standard}

Conflict of interest None.

Ethical approval Institutional Review Board approval was not required for this study.

Informed consent Informed consent was not applicable.

\section{References}

1. Sheetz KH, Norton EC, Dimick JB, Regenbogen SE (2019) Perioperative outcomes and trends in the use of robotic colectomy for medicare beneficiaries from 2010 through 2016. JAMA Surg 155:41

2. George EI, Brand TC, LaPorta A, Marescaux J, Satava RM (2018) Origins of robotic surgery: from skepticism to standard of care. J Soc Laparoendosc Surg 22(4):e201800039

3. Leal Ghezzi T, Campos Corleta O (2016) 30 Years of robotic surgery. World J Surg 40(10):2550-2557

4. Ramirez P, Frumovitz M, Pareja R, Lopez A, Vieira M, Ribeiro R, Buda A, Yan X, Shuzhong Y, Chetty N et al (2018) Minimally invasive versus abdominal radical hysterectomy for cervical cancer. N Engl J Med 379:1895

5. Lo BD, Leeds IL, Sundel MH, Gearhart S, Nisly G, Safar B, Atallah C, Fang SH (2020) Frailer patients undergoing robotic colectomies for colon cancer experience increased complication rates compared to open or laparoscopic approaches. Dis Colon Rectum 63:588-597

6. van Hilst J, de Rooij T, Bosscha K, Brinkman DJ, van Dieren S, Dijkgraaf MG, Gerhards MF, de Hingh IH, Karsten TM, Lips DJ et al (2019) Laparoscopic versus open pancreatoduodenectomy for pancreatic or periampullary tumours (LEOPARD-2): a multicentre, patient-blinded, randomised controlled phase 2/3 trial. Lancet Gastroenterol Hepatol 4(3):199-207

7. Spinoglio G, Bianchi PP, Marano A, Priora F, Lenti LM, Ravazzoni F, Petz W, Borin S, Ribero D, Formisano G et al (2018) Robotic versus laparoscopic right colectomy with complete mesocolic excision for the treatment of colon cancer: perioperative outcomes and 5-year survival in a consecutive series of 202 patients. Ann Surg Oncol 25(12):3580-3586

8. Crippa J, Grass F, Dozois EJ, Mathis KL, Merchea A, Colibaseanu DT, Kelley SR, Larson DW (2020) Robotic Surgery for Rectal Cancer Provides Advantageous Outcomes Over Laparoscopic Approach: Results From a Large Retrospective Cohort. Ann Surg. https://doi.org/10.1097/SLA.0000000000003805

9. Sheetz KH, Claflin J, Dimick JB (2020) Trends in the adoption of robotic surgery for common surgical procedures. JAMA Netw Open 3(1):e1918911

10. Ingraham AM, Richards KE, Hall BL, Ko CY (2010) Quality improvement in surgery: the American College of Surgeons National Surgical Quality Improvement Program approach. Adv Surg 44:251-267

11. Shiloach M, Frencher SK Jr, Steeger JE, Rowell KS, Bartzokis K, Tomeh MG, Richards KE, Ko CY, Hall BL (2010) Toward robust information: data quality and inter-rater reliability in the American College of Surgeons National Surgical Quality Improvement Program. J Am Coll Surg 210(1):6-16

12. Park JS, Choi GS, Park SY, Kim HJ, Ryuk JP (2012) Randomized clinical trial of robot-assisted versus standard laparoscopic right colectomy. Br J Surg 99(9):1219-1226

13. Waters PS, Cheung FP, Peacock O, Heriot AG, Warrier SK, O'Riordain DS, Pillinger S, Lynch AC, Stevenson ARL (2019) 
Successful patient-oriented surgical outcomes in robotic vs laparoscopic right hemicolectomy for cancer-a systematic review. Colorectal Dis 22(5):488-499

14. Ma S, Chen Y, Chen Y, Guo T, Yang X, Lu Y, Tian J, Cai H (2019) Short-term outcomes of robotic-assisted right colectomy compared with laparoscopic surgery: a systematic review and meta-analysis. Asian J Surg 42(5):589-598

15. Sheng S, Zhao T, Wang X (2018) Comparison of robot-assisted surgery, laparoscopic-assisted surgery, and open surgery for the treatment of colorectal cancer: a network meta-analysis. Medicine (Baltimore) 97(34):e11817

16. Jayne D, Pigazzi A, Marshall H, Croft J, Corrigan N, Copeland J, Quirke P, West N, Rautio T, Thomassen N et al (2017) Effect of robotic-assisted vs conventional laparoscopic surgery on risk of conversion to open laparotomy among patients undergoing resection for rectal cancer: the ROLARR randomized clinical trial. JAMA 318(16):1569-1580

17. Jimenez Rodriguez RM, Diaz Pavon JM, de La Portilla de Juan F, Prendes Sillero E, Hisnard Cadet Dussort JM, Padillo J (2011) Prospective randomised study: robotic-assisted versus conventional laparoscopic surgery in colorectal cancer resection. Cirugia espanola 89(7):432-438

18. Crippa J, Grass F, Achilli P, Mathis KL, Kelley SR, Merchea A, Colibaseanu DT, Larson DW (2020) Risk factors for conversion in laparoscopic and robotic rectal cancer surgery. Br J Surg 107:560
19. Li L, Jin J, Min S, Liu D, Liu L (2017) Compliance with the enhanced recovery after surgery protocol and prognosis after colorectal cancer surgery: a prospective cohort study. Oncotarget 8(32):53531-53541

20. Roulin D, Donadini A, Gander S (2015) The impact of enhanced recovery protocol compliance on elective colorectal cancer resection: results from an international registry. Ann Surg 261(6):1153-1159

21. Bach C, Miernik A, Schonthaler M (2014) Training in robotics: the learning curve and contemporary concepts in training. Arab J Urol 12(1):58-61

22. Sridhar AN, Briggs TP, Kelly JD, Nathan S (2017) Training in robotic surgery-an overview. Curr Urol Rep 18(8):58

23. Mark Knab L, Zenati MS, Khodakov A, Rice M, Al-Abbas A, Bartlett DL, Zureikat AH, Zeh HJ, Hogg ME (2018) Evolution of a novel robotic training curriculum in a complex general surgical oncology fellowship. Ann Surg Oncol 25(12):3445-3452

24. Moit H, Dwyer A, De Sutter M, Heinzel S, Crawford D (2019) A standardized robotic training curriculum in a general surgery program. JSLS J Soc Laparoendosc Surg 23(4):e201900045

Publisher's Note Springer Nature remains neutral with regard to jurisdictional claims in published maps and institutional affiliations. 